\title{
THE DEGREE OF COPOSITIVE APPROXIMATION BY POLYNOMIALS
}

\author{
D. LEVIATAN
}

\begin{abstract}
Jackson type theorems are established for the approximation of a function $f$ that changes sign finitely many times in $[-1,1]$ by polynomials $p_{n}$ which are copositive with it (i.e. $f p_{n} \geqslant 0$ on $[-1,1]$ ). The results yield the rate of nonconstrained approximation and are thus best possible in the same sense as in the nonconstrained case.
\end{abstract}

1. Introduction. In the past decade there has been a lot of activity on the question of copositive polynomial approximation and related topics. Given a continuous function $f$ in $[-1,1]$ which alternates in sign finitely many times in that interval, we will discuss the degree of approximation to it by polynomials that are copositive with it, i.e., polynomials $p_{n}$ so that $f(x) \cdot p_{n}(x) \geqslant 0$ for all $-1 \leqslant x \leqslant 1$.

In [3] Passow and Raymon state the following

THEOREM A. If $f \in C[-1,1]$ is proper piecewise monotone with nonvanishing peaks, then there is a constant $d$ depending on $f$, but not on $n$, such that for $n$ sufficiently large,

$$
\bar{E}_{n}(f) \leqslant d \omega(f, 1 / n) \text {, }
$$

where $\bar{E}_{n}(f)$ denotes the degree of copositive approximation to $f$ by polynomials of degree $\leqslant n$, and $\omega(f, \cdot)$ is the usual modulus of continuity of $f$.

Later, in [4], Roulier replaced the condition of proper piecewise monotonicity by a condition that $f$ is properly alternating, and proved the following result which improved Theorem $\mathbf{A}$ in many cases.

THEOREM B. If $f$ is properly alternating in $[-1,1]$, then there exists a constant $d$ depending on $f$, but not on $n$, such that for $n$ sufficiently large,

$$
\bar{E}_{n}(f) \leqslant d\left[\omega\left(f, 6 E_{n}(f) \delta^{-1}\right)+E_{n}(f)\right],
$$

where $E_{n}(f)$ is the degree of unconstrained approximation to $f$ by polynomials of degree $\leqslant n$, and $\delta>0$ is from the definition of proper alternation (see [4] for the definition).

The purpose of this paper is to obtain Jackson type estimates for the degree of copositive approximation of continuous functions with no restrictions of the above type and with an absolute constant (independent of $f$ ). Also we will get Jackson type estimates for copositive approximation of a differentiable function $f$, though the

Received by the editors May 21, 1982.

1980 Mathematics Subject Classification. Primary 41A29, 41A25; Secondary 41A10. 
constant in this case will depend on the location of some of the alternations and only holds for $n$ sufficiently large.

2. The main results. In view of the fact that the Jackson estimates are best possible, our first result is quite satisfactory and is much stonger than Theorem A.

THEOREM 1. There exists an absolute constant $C=C(r)$ such that for every $f \in C[-1,1]$ which alternates in sign $r$ times in $[-1,1], 0<r<\infty$, and each $n \geqslant 1$,

$$
\bar{E}_{n}(f) \leqslant C \omega(f, 1 / n) .
$$

When $f$ is differentiable we expect to get higher order Jackson estimates. $f$ changes sign at $-1<y<1$ if $f(y)=0$ and if for some $\varepsilon>0, f\left(x_{1}\right) f\left(x_{2}\right) \leqslant 0$ for all $y-\varepsilon<x_{1} \leqslant y \leqslant x_{2}<y+\varepsilon$. Such a $y$ is called an alternation point of $f$. If $f$ is continuously differentiable, then either $f^{\prime}(y)=0$ or $f^{\prime}(y) \neq 0$. In the latter case we will say that $y$ is a point of proper alternation. Our estimates will depend on the location of the points of proper alternation. How large $n$ should be will depend on $f$ (see the proof of Lemma 2).

Specifically we prove

ThEOREM 2. Let $f \in C^{j}[-1,1], 1 \leqslant j \leqslant 2$, alternate in sign $r$ times in $[-1,1]$ and let $-1<y_{1}<y_{2}<\cdots<y_{k}<1(k \leqslant r)$ be the points of proper alternation of $f$. Then for all $n$ sufficiently large,

$$
\bar{E}_{n}(f) \leqslant \frac{C}{n^{j}} \omega\left(f^{(j)}, \frac{1}{n}\right),
$$

where $C$ depends on $r$ and $y_{1}, \ldots, y_{k}$ but is otherwise independent of $f$ and $n$.

Although neither Theorem 1 nor Theorem 2 improve Theorem B directly, they yield, in our mind, more satisfactory estimates on the degree of copositive approximation by polynomials. In fact, in the proof of Theorem 2, we will make use of Lemma 2 of $\S 3$, which is a direct improvement of Theorem B. We are indebted to Professor Roulier for the statement and proof of that lemma.

3. Proofs. The proof of Theorem 1 follows immediately by the following lemma due to Beatson and the author [1]. The lemma is not stated in this form in the above paper but can be readily obtained by the proof of Theorem 1 there.

LEMMA 1. Let $g \in C^{1}[-1,1]$ be piecewise monotone (change monotonicity $r$ times, $0<r<\infty)$ in $[-1,1]$. Then for each $n \geqslant 1$ there is a polynomial $p_{n}$ comonotone with $g$ such that

$$
\left\|g^{(i)}-p_{n}^{(i)}\right\| \leqslant C n^{i-1} \omega\left(g^{\prime}, 1 / n\right), \quad i=0,1 .
$$

$C=C(r)$ is an absolute constant independent of $g$ and $n$.

Evidently Theorem 1 follows by setting $g(x)=\int^{x} f$, whereupon $g$ is piecewise monotone and $p_{n}^{\prime}$ is copositive with $f$. Now (1) follows from (3) with $i=1$.

For the proof of Theorem 2 we need the following lemma due to Roulier. This lemma improves the corollary in [4]. 
Lemma 2. Let $f \in C^{1}[-1,1]$ alternate in sign finitely many times in $[-1,1]$ and assume that all alternation points are points of proper alternation. Then for all $n$ sufficiently large,

$$
\bar{E}_{n}(f) \leqslant C E_{n}(f)
$$

where $C$ depends on the alternation points but is otherwise independent of $f$ and $n$.

Proof. Let $-1<y_{1} \cdots<y_{r}<1$ be the alternation points. Then $\left|f^{\prime}\left(y_{i}\right)\right|>0$ so that there is an $\varepsilon>0$ such that $\left|f^{\prime}(x)\right|>2 \delta>0$ for all $x \in \cup_{i=1}^{r}\left(y_{i}-\varepsilon, y_{i}+\varepsilon\right)$. Since $f \in C^{1}[-1,1]$ if $P_{n}$ is the $n$th polynomial of best approximation of $f$, then

$$
\left\|f-P_{n}\right\|_{[-1,1]}=o\left(n^{-1}\right), \quad \text { as } n \rightarrow \infty .
$$

Hence it follows by the lemma in [5] that if $-1<a<b<1$, then

$$
\left\|f^{\prime}-P_{n}^{\prime}\right\|_{[a, b]}=o(1), \quad \text { as } n \rightarrow \infty,
$$

where the $o$ depends only on $a$ and $b$. Thus (choosing $\varepsilon, a$ and $b$ properly) we see that for $n$ sufficiently large,

$$
\left|P_{n}^{\prime}(x)\right|>\delta \text { for } x \in \bigcup_{i=1}^{r}\left(y_{i}-\varepsilon, y_{i}+\varepsilon\right) \text { for some } \varepsilon>0,
$$

and $f^{\prime}(x) \cdot P_{n}^{\prime}(x)>0$ for all $x \in \bigcup_{i=1}^{r}\left(y_{i}-\varepsilon, y_{i}+\varepsilon\right)$. Now we add a correction to ensure that $y_{i}$ be alternation points for the approximating polynomials. Let $q_{r} \in \pi_{r}$ be the Lagrange interpolation to $-P_{n}$ at the $y_{i}$ 's, that is,

$$
q_{r}\left(y_{i}\right)=-P_{n}\left(y_{i}\right), \quad i=1, \ldots, r .
$$

Then

$$
\left\|q_{r}\right\| \leqslant B \max _{1 \leqslant i \leqslant r}\left|P_{n}\left(y_{i}\right)\right|=B \max _{1 \leqslant i \leqslant r}\left|f\left(y_{i}\right)-P_{n}\left(y_{i}\right)\right| \leqslant B\left\|f-P_{n}\right\|=B E_{n}(f),
$$

where $B$ depends only on the $y_{i}$ 's. By Markov's inequality,

$$
\left\|q_{r}^{\prime}\right\| \leqslant B r^{2} E_{n}(f) \rightarrow 0, \quad \text { as } n \rightarrow 0 .
$$

Together with (5) this implies for $n$ sufficiently large that the polynomial $Q_{n}(x)=$ $P_{n}(x)+q_{r}(x)$ is copositive with $f$ on the intervals $\left(y_{i}-\varepsilon, y_{i}+\varepsilon\right), i=1, \ldots, r$. Define $h_{r}(x)= \pm\left(x-y_{1}\right) \cdots\left(x-y_{r}\right)$ where the sign is chosen so that $h_{r}$ and $f$ are copositive, and let

$$
\rho=\min \left\{\left|h_{r}(x)\right|: x \in[-1,1] \backslash \bigcup_{i=1}^{r}\left(y_{i}-\varepsilon, y_{i}+\varepsilon\right)\right\} .
$$

Set

$$
p_{n}(x)=Q_{n}(x)+(2+B) \rho^{-1} E_{n}(f) h_{r}(x) .
$$

Then it is readily seen that $f$ and $p_{n}$ are copositive and

$$
\left\|f-p_{n}\right\| \leqslant E_{n}(f)+B E_{n}(f)+\frac{2+B}{\rho}\left\|h_{r}\right\| E_{n}(f)=C E_{n}(f) .
$$

Thus (4) is established. 
Proof of Theorem 2. We will prove Theorem 2 by induction on the number of alternation points that are not points of proper alternation. If there are none, then (2) follows by virtue of Lemma 2 and Jackson's Theorem. Suppose we have established (2) for functions with $s \geqslant 0$ nonproper alternations and $f$ has $s+1$ nonproper alternations. We may assume that $x=0$ is one of these points. (This assumption makes the writing simpler.) Otherwise if the nonproper alternation is at $x=\alpha$, one has to construct a polynomial which mimics $\operatorname{sgn}(x)$ on $[-2,2]$ and then substitute in it $x-\alpha$ for $x$. (Compare with (8) later in the proof.) Now define the flipped over function

$$
\hat{f}(x)= \begin{cases}-f(x), & -1 \leqslant x \leqslant 0 \\ f(x), & 0<x \leqslant 1\end{cases}
$$

Since $f^{\prime}(0)=0$ it follows that if $f \in C^{2}[-1,1]$, then $f^{\prime \prime}(0)=0$.

Thus $\hat{f} \in C^{j}[-1,1], 1 \leqslant j \leqslant 2$, and

$$
\omega\left(\hat{f}^{(j)}, \cdot\right) \leqslant 2 \omega\left(f^{(j)}, \cdot\right) .
$$

Also $\hat{f}$ has $s$ nonproper alternations so, by virtue of (6) and the induction assumption, there exists a polynomial $q_{n}$ copositive with $\hat{f}$ such that

$$
\left\|\hat{f}-q_{n}\right\| \leqslant \frac{C}{n^{j}} \omega\left(f^{(j)}, \frac{1}{n}\right) .
$$

DeVore [2, p. 908] has shown that it is possible to construct a polynomial $t_{n}(x)$ which mimics the function $\operatorname{sgn}(x)$. Specifically $t_{n}$ is odd and increasing in $[-1,1]$, $t_{n}(1)=1$, and

$$
\left|\operatorname{sgn}(x)-t_{n}(x)\right| \leqslant A|n x|^{-3}, \quad x \in[-1,0) \cup(0,1] .
$$

Evidently the polynomial $p_{n}=q_{n} t_{n}$ is copositive with $f$ and

$$
\begin{aligned}
f(x)-p_{n}(x) & =\left[\hat{f}(x)-q_{n}(x)\right] \operatorname{sgn}(x)+q_{n}(x)\left[\operatorname{sgn}(x)-t_{n}(x)\right] \\
& =I_{1}+I_{2}, \quad \text { say. }
\end{aligned}
$$

By (7)

$$
\left|I_{1}\right| \leqslant \frac{C}{n^{j}} \omega\left(f^{(j)}, \frac{1}{n}\right) .
$$

Notice that for $\hat{f} \in C^{j}[-1,1]$ with $\hat{f}(0)=\cdots=\hat{f}^{(j)}(0)=0$ we have

$$
\begin{aligned}
|\hat{f}(x)| & =|\hat{f}(x)-\hat{f}(0)|=|x|\left|\hat{f}^{\prime}(\theta x)-\hat{f}^{\prime}(0)\right| \\
& \leqslant \cdots \leqslant\left|x \psi^{j}\right| \hat{f}^{(j)}\left(\theta_{1} x\right)-\hat{f}^{(j)}(0)|\leqslant| x \psi_{\omega} \omega\left(f^{(j)},|x|\right) .
\end{aligned}
$$

Hence for $|x|<1 / n$ it follows by (6) and (7) that

$$
\left|q_{n}(x)\right| \leqslant\left\|q_{n}-\hat{f}\right\|+|\hat{f}(x)| \leqslant \frac{B}{n^{j}} \omega\left(f^{(j)}, \frac{1}{n}\right),
$$

which, together with $\left|\operatorname{sgn}(x)-t_{n}(x)\right| \leqslant 1$, implies

$$
\left|I_{2}\right| \leqslant \frac{B}{n^{j}} \omega\left(f^{(j)}, \frac{1}{n}\right) \text {. }
$$


If $|x| \geqslant 1 / n$, let $i / n \leqslant|x|<(i+1) / n$ where $1 \leqslant i \leqslant n$. Then it follows by virtue of (6), (7), (8) and (10) that

$$
\begin{aligned}
\left|I_{2}\right| \leqslant \mid \hat{f}(x) & -q_{n}(x)|+| \hat{f}(x)|| \operatorname{sgn}(x)-t_{n}(x) \mid \\
& \leqslant \frac{C}{n^{j}} \omega\left(f^{(j)}, \frac{1}{n}\right)+A|n x|^{-3}|x| \omega\left(\hat{f}^{(j)},|x|\right) \quad(\text { recall that } 1 \leqslant j \leqslant 2) \\
& \leqslant \frac{C}{n^{j}} \omega\left(f^{(j)}, \frac{1}{n}\right)+\frac{4 A}{n^{j}} \frac{i+1}{i^{3-j}} \omega\left(f^{(j)}, \frac{1}{n}\right)=\frac{D}{n^{j}} \omega\left(f^{(j)}, \frac{1}{n}\right) .
\end{aligned}
$$

Combining this with (9) and (11) establishes our theorem.

\section{REFERENCES}

1. R. K. Beatson and D. Leviatan, On comonotone approximation, Canad. Math. Bull. (to appear).

2. R. A. DeVore, Monotone approximation by polynomials, SIAM J. Math. Anal. 8 (1977), 906-921.

3. E. Passow and L. Raymon, Copositive polynomial approximation, J. Approx. Theory 12 (1974), 299-304.

4. J. A. Roulier, The degree of copositive approximation, J. Approx. Theory 19 (1977), 253-258.

5 . , Nearly comonotone approximation, Proc. Amer. Math. Soc. 47 (1975), 84-88.

Department of Mathematics, University of Connecticut, Storrs, ConNecticut 06268

Current address: Department of Mathematics, Tel-Aviv University, Ramat Aviv, Israel 